\title{
Certified, Efficient and Sharp Univariate Taylor Models in COQ
}

\author{
Érik Martin-Dorel, Laurence Rideau, \\ and Laurent Théry \\ Inria Sophia Antipolis \\ 2004 route des Lucioles, BP 93 \\ 06902 Sophia Antipolis Cedex, France \\ Email: erik.martin-dorel@ens-lyon.org \\ Email: laurence.rideau@inria.fr \\ Email: laurent.thery@inria.fr
}

\author{
Micaela Mayero \\ Université Paris 13 \\ LIPN (UMR CNRS 7030), Institut Galilée \\ 99 avenue Jean-Baptiste Clément \\ 93430 Villetaneuse, France \\ Ioana Paşca \\ Université Montpellier 2 \\ IUT de Nîmes \\ 8 rue Jules Raimu \\ Email: micaela.mayero@lipn.univ-paris13.fr Email: ioana.pasca@iut-nimes.fr
}

\begin{abstract}
We present a formalisation, within the COQ proof assistant, of univariate Taylor models. This formalisation being executable, we get a generic library whose correctness has been formally proved and with which one can effectively compute rigorous and sharp approximations of univariate functions composed of usual functions such as $1 / x, \sqrt{x}, e^{x}, \sin x$ among others. In this paper, we present the key parts of the formalisation and we evaluate the quality of our certified library on a set of examples.
\end{abstract}

\section{Introduction AND Motivations}

Polynomial approximations are a practical way to represent real-valued functions. In fact, on processors, where the only primitive arithmetic operations are,+- , and $\times$, they are the only effective way to compute real-valued functions. The quality of the approximation naturally comes into play: being able to guarantee some bounds on the error that occurs when using the approximation instead of the real function is mandatory for the reliability of numerical software. Yet bounds are not always available and when so they are often very difficult to be proved formally.

The work presented here addresses this issue of reliability. We provide a systematic way to formally prove the error bounds of some specific polynomial approximations. This is done in the COQ system but the same approach could be implemented in any other proof assistant. Our starting point is the notion of rigorous polynomial approximations (RPAs), which consists of pairs $(P, \boldsymbol{\Delta})$ where $P$ is a polynomial in a given basis and $\boldsymbol{\Delta}$ an interval error bound. Several symbolic-numeric techniques that rely on such a data type have been presented in [1]. They heavily rely on interval arithmetic. For instance, most of these algorithms manipulate polynomials with tight interval coefficients. Also, rounding errors that may occur when computing the polynomial approximation can easily be handled in this setting. In the following, we will especially focus on RPAs in the Taylor polynomial basis, which we will refer to as Taylor models (TMs), borrowing the term coined by Berz and Makino [2], [3].

This research was partly funded by the TaMaDi project of the Agence Nationale de la Recherche (ref. ANR-2010-BLAN-0203-01).
We formalise univariate Taylor models in the COQ proof assistant, aiming at:

- Genericity: the implementation should be extensible and applicable to a large class of problems such as floating-point implementations of elementary functions, numerical quadrature, or ordinary differential equation (ODE) solving;

- Efficiency: computing the approximations (even if performed in a trusted environment with restricted computing power) should be reasonably fast;

- Correctness: no underestimate is possible, the computed error-bounds should be proved correct;

- Sharpness: the algorithms should lead (most of the time) to sharp bounds.

A preliminary version of this work was already presented in [4]. The main achievement of what is presented here is that, now, a correctness proof is attached to each of our algorithms. Also, some major improvements in the algorithms have been made in order to get tighter error bounds for basic functions as well as for the division of Taylor models.

Our implementation of Taylor models is composed of a set of models for basic functions (exp, $\sin , \sqrt{\cdot}$, and others) and another set of algorithms that are used to combine these models (for addition, multiplication, composition, or division of two functions). We start, in Section II, by describing the general framework of Taylor models as well as the formal tools that are needed for their implementation and proof. In Section III, we present in detail the issues related to Taylor models for basic functions. We then present Taylor models for composite functions in Section IV. In Section V, we provide some benchmarks. Finally, Section VI relates our work with other approaches. 


\section{Generic TAYlor Models AND Their FORMALISATION}

Given a point $x_{0}$ and an interval ${ }^{1} \boldsymbol{I}$ around this point, a Taylor model usually consists of a pair $(P, \boldsymbol{\Delta})$ where $P$ is a polynomial in the Taylor basis around $x_{0}$ and $\boldsymbol{\Delta}$ is an interval. A Taylor model approximates a whole set of functions : the functions that are at a distance of less than $\boldsymbol{\Delta}$ over $\boldsymbol{I}$. More formally, $(P, \boldsymbol{\Delta})$ approximates $f$ if and only if $\forall x \in \boldsymbol{I}, f(x)-P(x) \in \boldsymbol{\Delta}$. Starting from a given function $f$, a natural way to derive a Taylor model $(P, \boldsymbol{\Delta})$ is to use the Taylor-Lagrange formula

Theorem 1: If $f$ is a real-valued function that is $n+1$ times derivable on an interval $\boldsymbol{I}$ and $x_{0}$ is a point of $\boldsymbol{I}$ then we can consider the $n^{\text {th }}$-order Taylor expansion of $f$ around $x_{0}$. For all $x$ in $\boldsymbol{I}$, there exists a $\xi$ between $x_{0}$ and $x$ such that

$f(x)=\underbrace{\left(\sum_{i=0}^{n} \frac{f^{(i)}\left(x_{0}\right)}{i !}\left(x-x_{0}\right)^{i}\right)}_{P(x)}+\underbrace{\frac{f^{(n+1)}(\xi)}{(n+1) !}\left(x-x_{0}\right)^{n+1}}_{\Delta\left(x_{0}, x, \xi\right)}$.

If $f^{(n+1)}(x)$ can be bounded over the interval $\boldsymbol{I}$, it is then easy to compute an interval $\boldsymbol{\Delta}$ so that $\Delta\left(x_{0}, x, \xi\right) \in \boldsymbol{\Delta}$ for $x \in \boldsymbol{I}$ and build the approximation $(P, \boldsymbol{\Delta})$. Doing so, $\boldsymbol{\Delta}$ accumulates the "method error". Note that, in practice, it is not always possible to compute an approximation with exact numbers. The coefficients of the polynomial need to be rounded to rational numbers, or even more restrictively to floating-point numbers. This is not a problem for Taylor models since rounding errors can be incorporated into the $\boldsymbol{\Delta}$. The approximation is then less accurate but still valid.

As explained in the introduction, a key ingredient to get effective Taylor models is to use interval arithmetic. In this setting, the polynomial of a Taylor model has tight interval coefficients. As usual, we represent polynomials as lists of coefficients, but we do not enforce that the last coefficient is non-zero. In the following, we will then talk about the "order" of a Taylor model rather than its "degree" and reason in terms of the size of the corresponding list of coefficients. An $n^{\text {th }}$-order Taylor model $(\boldsymbol{P}, \boldsymbol{\Delta})$ will have size $\boldsymbol{P}=n+1$. Moreover, the "expansion point" will not be a point $x_{0}$ but rather a small interval $\boldsymbol{x}_{\mathbf{0}}$. Taylor models can thus be built around an irrational expansion point. The definition of validity is then rephrased as

Definition 1: $(\boldsymbol{P}, \boldsymbol{\Delta})$ is a valid TM for $f: \mathbb{R} \rightarrow \mathbb{R}$ over $\boldsymbol{I}$ around $\boldsymbol{x}_{\mathbf{0}}$ if we have $\boldsymbol{x}_{\mathbf{0}} \subset \boldsymbol{I}, 0 \in \boldsymbol{\Delta}$, and for all $\xi_{0} \in \boldsymbol{x}_{\mathbf{0}}$, there exists a polynomial $Q$ over $\mathbb{R}$ such that

$$
\left\{\begin{array}{l}
\operatorname{size} Q=\operatorname{size} \boldsymbol{P}, \\
\forall k<\operatorname{size} \boldsymbol{P}, \quad Q_{k} \in \boldsymbol{P}_{k}, \\
\forall x \in \boldsymbol{I}, \quad f(x)-\sum_{i<\operatorname{size} Q} Q_{i} \cdot\left(x-\xi_{0}\right)^{i} \in \boldsymbol{\Delta} .
\end{array}\right.
$$

Formalising this definition in COQ is straightforward. For the polynomials, we use the library provided by the SSREFLECT extension [5]. For the intervals, we use the Coq.Interval library [6] that provides abstract data types

\footnotetext{
${ }^{1}$ Intervals (as well as polynomials with interval coefficients) will be printed in bold.
}

Table I. Semantics of the InduCtive type For Intervals

\begin{tabular}{l|l} 
COQ term & mathematical meaning \\
\hline Ibnd (Xreal $x)($ Xreal $y)$ with $x>y$ & $\varnothing$ \\
\hline Ibnd (Xreal $x$ ) Xreal $y$ ) with $x \leqslant y$ & {$[x, y]$} \\
\hline Ibnd (Xreal $x$ ) Xnan & {$[x,+\infty[$} \\
\hline Ibnd Xnan (Xreal $y$ ) & ]$-\infty, y]$ \\
\hline Ibnd Xnan Xnan & $\mathbb{R}$ \\
\hline Inan & $\mathbb{R} \cup\{\mathrm{NaN}\}$
\end{tabular}

for an interval arithmetic that handles undefined values (NaNs):

$\overline{\mathbb{R}}:=$ Xnan $\mid$ Xreal $(r: \mathbb{R})$.

interval $:=$ Inan $\mid$ Ibnd $(1 \mathrm{u}: \overline{\mathbb{R}})$.

The semantics of these "NaN" values is summarised in Table I. Note that, in this context, we have Xnan $\in$ Inan but Xnan $\notin$ (Ibnd Xnan Xnan). The definition i_validTM of the formal development that is available $\mathrm{at}^{2}$

\section{http://tamadi.gforge.inria.fr/CoqApprox/}

is a direct transcription of Definition 1. The only main difference is that the function $f$ is lifted from $\mathbb{R} \rightarrow \mathbb{R}$ to $\overline{\mathbb{R}} \rightarrow \overline{\mathbb{R}}$, and the coefficients of $Q$ are not in $\mathbb{R}$, but in $\overline{\mathbb{R}}$.

The type $\mathbb{R}$ in COQ is defined via a classical axiomatisation of an Archimedean ordered complete field [7] in the Reals standard library. It provides all the basic theorems usually involved in real analysis, e.g., about differentials or integrals, but no computation is available.

The SSREFLECT [5] extension provides its own tactic language and libraries, and has been designed to make it easier to formalise mathematics. In this work, two libraries are of special interest. A first one defines standard algebraic structures and the theorems associated to them. A second one provides a very powerful theory bigop for iterated "big" operations like summations. For polynomial approximations over $\overline{\mathbb{R}}$, the theory bigop was quite handy to use, except that we had to circumvent the fact that the multiplication of $\overline{\mathbb{R}}$ is not distributive over the addition, since (Xreal 0) is not a "cancelling element" for the multiplication. This issue is directly related to the presence of undefined values such as Xnan and Inan in the formalism of Coq.Interval. Some careful case analyses are then frequently required when developing our correctness proofs.

The Coq.Interval library also provides ways to instantiate intervals and in particular to get intervals we can compute with. One such instantiation uses two floatingpoint numbers to represent the bounds of the interval. The floating-point numbers are also defined within Coq.Interval either by pairs of integers (a mantissa and an exponent) or by an undefined value :

float $:=$ Fnan | Float $(m e: \mathbb{Z})$.

The operations on these numbers are defined in Coq.Interval (such as,,$+- \times, \div, \sqrt{ } \cdot$, comparison, etc.) but they do not assume any bound on the exponent. Yet they take a precision argument that is used for rounding

\footnotetext{
${ }^{2}$ In the following, we will frequently refer to the COQ code available at this URL.
} 
the mantissa of the result. Using such an instantiation, it is then possible to specialise the previous definition that uses intervals to polynomials with simple floating-point coefficients, as explained below.

From any pair $(\boldsymbol{P}, \boldsymbol{\Delta})$ that is a valid TM according to Definition 1 , we can easily produce a pair $\left(P, \boldsymbol{\Delta}^{\prime}\right)$ where $P$ is polynomial with floating-point coefficients satisfying:

Definition 2: $\left(P, \boldsymbol{\Delta}^{\prime}\right)$ is a valid TM for $f: \overline{\mathbb{R}} \rightarrow \overline{\mathbb{R}}$ over $\boldsymbol{I}$ around $\boldsymbol{x}_{\mathbf{0}}$ if we have $\boldsymbol{x}_{\mathbf{0}} \subset \boldsymbol{I}, 0 \in \boldsymbol{\Delta}^{\prime}$, and

$\forall \xi_{0} \in \boldsymbol{x}_{\mathbf{0}}, \quad \forall x \in \boldsymbol{I}, \quad f(x)-\sum_{i<\text { size } P} P_{i} \cdot\left(x-\xi_{0}\right)^{i} \in \boldsymbol{\Delta}^{\prime}$.

It suffices to take the midpoint of each interval coefficient of $\boldsymbol{P}$ and add the corresponding errors to $\boldsymbol{\Delta}$. In the formal development, Definition 2 is formalised as a predicate $f_{\text {_validTM, }}$ and the function i2f_tm transforms $(\boldsymbol{P}, \boldsymbol{\Delta})$ into $\left(P, \boldsymbol{\Delta}^{\prime}\right)$.

Generic implementation of polynomials, coefficients and intervals: As more detailed in [4] and in [8], COQ provides three mechanisms for modularisation: type classes, structures, and modules. Modules are less generic than the other two (which are first-class citizens) but they have a better computational behaviour: module applications are performed statically, so the code that is executed is often more compact. As our generic implementation only requires simple parametricity, we have been using modules only. First, abstract interfaces called Module Types are defined. Then concrete "instances" of these abstract interfaces are created by providing an implementation for all the fields of the Module Type. The definition of Modules can be parameterised by other Modules. These parameterised modules are crucial to factorise code in our structures.

We describe abstract interfaces for polynomials and for their coefficients in the form of Module Types. The interface for coefficients contains the common base of all kinds of "computable real numbers" we may want to use. Usually coefficients of a polynomial are taken in a ring. We cannot do this here. For example, addition of two intervals with floating-point bounds is not associative. Therefore, our abstract interface for coefficients only contains the required operations (addition, multiplication, etc.). Other basic properties (associativity, distributivity, etc.) are ruled out. The case of abstract polynomials is similar. They are also a Module Type but this time parameterised by the coefficients. The interface only contains the operations on polynomials (addition, evaluation, iterator, etc.) along with the properties that are satisfied by all common instantiations of polynomials. Finally for intervals, we directly use the abstract interface provided by the Coq.Interval library.

We are now able to give the definition of our RPA.

Module RigPolyApprox

(C: BaseOps)(Pol: PolyOps C) (I: IntervalOps). Record rpa := RPA $\{$ approx: Pol.T; error: I.type $\}$.

The module is parameterised by $\mathrm{C}$ (the coefficients), by Pol (the polynomials with coefficients in C), and by I (the intervals). A rpa structure consists of a polynomial approx and an interval error. Taylor models will be defined as an instance of the generic rpa structure.

\section{TAYLOR MODELS FOR BASIC FunCtions}

\section{A. Mathematical setup}

Relying on Theorem 1, it suffices to take an interval enclosure of $\Delta\left(x_{0}, x, \xi\right)$ with respect to $x_{0} \in \boldsymbol{x}_{\mathbf{0}}, x \in \boldsymbol{I}$ and $\xi \in \boldsymbol{I}$ to get a value for $\boldsymbol{\Delta}$. Yet this strategy would yield very pessimistic error bounds for composite functions such as $x \mapsto e^{1 / \cos x}[9]$. Hence the interest to use the following two-level strategy:

- For each basic function $\left(\sqrt{x}, e^{x}, \sin x\right.$, etc. $)$ involved in (the leafs of) the expression tree of $f(x)$, compute a Taylor model using, for instance, a naive enclosure of the Taylor-Lagrange remainder;

- Combine the various Taylor models computed for the atoms of expression $f(x)$ using the algebraic rules that correspond to operations,$+ \times$, as well as $\circ$ (composition).

For example, to compute a Taylor model for $x \mapsto e^{1 / \cos x}$ we can notice that $e^{1 / \cos x}=$ $\exp \circ\left(\left(x \mapsto \frac{1}{x}\right) \circ \cos \right)(x)$ and first compute a Taylor model for $\cos (x)$, then deduce a Taylor model for the overall function by using twice the algebraic rule for composition. We will give more details on these rules in Section IV.

\section{B. Formal setup}

In this section we give some insight on our formalisation of Taylor models for basic functions. The focus is on the design of the generic algorithm and its correctness proof.

A first building block is composed by the formalisation of Theorem 1. This is the topic of the upcoming Section III-B1. Section III-B2 is devoted to the formalisation of an efficient computation of Taylor polynomials. Section III-B3 focuses on the generic computation of sharp error bounds for these polynomials. Finally, Section III-B4 deals with the specialisation of our generic framework to concrete functions.

1) Formal proof of the Taylor-Lagrange theorem: As the Taylor-Lagrange theorem (Theorem 1) was not available in the Reals standard library of COQ, our first task has been to prove this result using the mean value theorem. An important choice in this formal proof is the formalisation of higher-order derivatives for a given function. In the Reals library, we can only talk about the derivative of a function if we have a proof that the function is actually derivable. This is often annoying when doing proofs involving derivatives. For the Taylor-Lagrange theorem and in order to talk about the $n^{\text {th }}$-order derivative of a given function $f$, we consider a function $D: \mathbb{N} \rightarrow \mathbb{R} \rightarrow \mathbb{R}$ which has the following properties:

$$
\left\{\begin{array}{l}
D_{0}=f \\
\forall k<n, D_{k} \text { is derivable and its derivative is } D_{k+1}
\end{array}\right.
$$


Since in our formalisation we use real numbers extended with a NaN value, we had to provide a specific version of the Taylor-Lagrange theorem for this type $\overline{\mathbb{R}}$. The advantage is that we can more easily talk about derivatives. We need not to worry whether a function is derivable at a point or not. If not, the derivative simply takes the value Xnan at this point. The disadvantage is that we need to perform a careful case analysis in the proof to cover all potential Xnan values.

2) Efficient computation of Taylor polynomials for the class of D-finite functions: In order to implement Taylor models for basic functions in a generic way, we consider a large class of functions whose successive derivatives can be computed in a uniform and efficient way. We thus focus on the so-called $D$-finite functions. They correspond to solutions of homogeneous linear ordinary differential equations (LODEs) with polynomial coefficients, that is equations of the form

$$
a_{r}(x) y^{(r)}(x)+\cdots+a_{1} y^{\prime}(x)+a_{0}(x) y(x)=0,
$$

where the $a_{k}$ are univariate polynomials over a given field. Most common functions are $D$-finite, while a simple counter-example is tan. A nice feature of this class of functions is that the Taylor series coefficients of any $D$ finite function satisfy a linear recurrence relation.

We thus provide the functions trec1, trec2 and trecN that allow one to compute polynomials whose coefficients are given by a first order, second order, or $N^{t h}$-order recurrence along with the appropriate number of initial conditions. Having specific functions for recurrences of small order makes it possible to have an optimised implementations for these frequent cases. For instance, trec1 is a tail-recursive function that has type

trec1: $(\mathrm{T} \rightarrow \mathbb{N} \rightarrow \mathrm{T}) \rightarrow \mathrm{T} \rightarrow \mathbb{N} \rightarrow$ Pol.T

and produces the polynomial $\operatorname{trec} 1\left(G, c_{0}, n\right)=\sum_{i=0}^{n} c_{i} X^{i}$ where $c_{n}=G\left(c_{n-1}, n\right)$ for all $n>1$.

3) Zumkeller's technique and sharp error bounds: Let $f$ be a basic function, and assume that we have a function $\boldsymbol{T}: \mathrm{T} \rightarrow \mathbb{N} \rightarrow$ Pol.T (possibly based on the $D$-finite recurrences presented in the previous section) to compute the $n^{\text {th }}$-order Taylor polynomial of $f$ around a given point. Then we can compute a Taylor model of $f$ by using Algorithm 1 below.

Algorithm 1 (Zumkeller's technique):

Input: $\boldsymbol{F}$ : interval evaluator for function $f$

Input: $\boldsymbol{T}\left(\boldsymbol{y}_{\mathbf{0}}, n\right): n^{\text {th }}$-order Taylor polynomial around $\boldsymbol{y}_{\mathbf{0}}$

Input: $\boldsymbol{x}_{\mathbf{0}} \subset \boldsymbol{I}$ and $n \in \mathbb{N}$

Output: $(P, \Delta)$

1: $\boldsymbol{P} \leftarrow \boldsymbol{T}\left(\boldsymbol{x}_{0}, n\right)$

2: $\boldsymbol{\Gamma} \leftarrow\left[X^{n+1}\right] \boldsymbol{T}(\boldsymbol{I}, n+1)$

3: if $(\sup \boldsymbol{\Gamma} \leqslant 0$ or $\inf \boldsymbol{\Gamma} \geqslant 0)$ and $\boldsymbol{I}$ is bounded then

4: $\quad \boldsymbol{a} \leftarrow[\inf \boldsymbol{I}, \inf \boldsymbol{I}]$

5: $\quad \boldsymbol{b} \leftarrow[\sup \boldsymbol{I}, \sup \boldsymbol{I}]$

6: $\quad \boldsymbol{\Delta}_{a} \leftarrow \boldsymbol{F}(\boldsymbol{a})-\boldsymbol{P}\left(a-\boldsymbol{x}_{0}\right)$

7: $\quad \boldsymbol{\Delta}_{\boldsymbol{b}} \leftarrow \boldsymbol{F}(\boldsymbol{b})-\boldsymbol{P}\left(\boldsymbol{b}-\boldsymbol{x}_{\mathbf{0}}\right)$

8: $\quad \boldsymbol{\Delta}_{\boldsymbol{x}_{\mathbf{0}}} \leftarrow \boldsymbol{F}\left(\boldsymbol{x}_{\mathbf{0}}\right)-\boldsymbol{P}\left(\boldsymbol{x}_{\mathbf{0}}-\boldsymbol{x}_{\mathbf{0}}\right)$

9: $\quad \boldsymbol{\Delta} \leftarrow \boldsymbol{\Delta}_{a} \vee \boldsymbol{\Delta}_{\boldsymbol{b}} \vee \boldsymbol{\Delta}_{x_{0}}$

10: else
11: $\boldsymbol{\Delta} \leftarrow \boldsymbol{\Gamma} \times\left(\boldsymbol{I}-\boldsymbol{x}_{\mathbf{0}}\right)^{n+1}$

12: end if

The computation of $\boldsymbol{P}$ is straightforward but the one of $\boldsymbol{\Delta}$ deserves some detailed explanation.

To start with, the else branch computes a naive enclosure of the Taylor-Lagrange remainder. In particular, due to the very similar shape of this enclosure with respect to a Taylor coefficient of degree $(n+1)$ "around $\boldsymbol{I}$ ", we compute both this enclosure and the Taylor coefficients of $\boldsymbol{P}$ in a uniform way (cf. Line 1 as well as Line 2, where the notation $\left[X^{n}\right] Q$ denotes the coefficient of $Q$ of degree $n$ ).

Now let us focus on the then branch of Algorithm 1. Note that this part of the algorithm is new with respect to the implementation that we first presented in [4], and this optimisation computes sharper error bounds for basic functions, while being as generic. Indeed, it can be noted that the expressions $\boldsymbol{\Delta}_{\boldsymbol{x}_{\mathbf{0}}}, \boldsymbol{\Delta}_{\boldsymbol{a}}$ and $\boldsymbol{\Delta}_{\boldsymbol{b}}$ correspond to the evaluation of the Taylor-Lagrange remainder on the small intervals $\boldsymbol{x}_{\mathbf{0}}, \boldsymbol{a}$ and $\boldsymbol{b}$ (composed of the endpoints of $\boldsymbol{I}$ ). As a result, beyond the slight rounding errors that may occur in these evaluations, the value of $\boldsymbol{\Delta}$ that is obtained in this branch is the sharpest possible bound we could achieve. It then remains to ensure that this value is not underestimated.

We give below an overview of the main steps involved in its formal proof, which relies on the Proposition 2.2.1 in Mioara Joldeş' thesis [1], itself based on Lemma 5.12 in Roland Zumkeller's thesis [10]. Let us denote the TaylorLagrange remainder of $f$ by

$$
R_{n}\left(f, \xi_{0}\right)(x):=f(x)-\sum_{i=0}^{n} \frac{f^{(i)}\left(\xi_{0}\right)}{i !} \cdot\left(x-\xi_{0}\right)^{i} .
$$

We have successively proved the following steps in COQ.

- If the condition holds on Line 3, then $f^{(k)}(x)$ is never Xnan for $0 \leqslant k \leqslant n+1$ and $x \in \boldsymbol{I}$, and $f^{(n+1)}$ has a constant sign over $[\inf \boldsymbol{I}, \sup \boldsymbol{I}]$.

- We have $\forall \xi_{0} \in \boldsymbol{I}, \forall x \in \boldsymbol{I}, R_{n}\left(f, \xi_{0}\right)^{\prime}(x)=$ $R_{n-1}\left(f^{\prime}, \xi_{0}\right)(x)$, and, by Theorem 1, there exists $\xi^{\prime}$ between $\xi_{0}$ and $x$ such that $R_{n-1}\left(f^{\prime}, \xi_{0}\right)(x)=$ $\frac{\left(f^{\prime}\right)^{(n)}\left(\xi^{\prime}\right)}{n !}\left(x-\xi_{0}\right)^{n}$ (the case where $n=0$ is handled separately in the formalisation).

- Then we study the sign of the expression $R_{n}\left(f, \xi_{0}\right)^{\prime}(x)$ to conclude that $R_{n}\left(f, \xi_{0}\right)$ is monotonous over $\left[\inf \boldsymbol{I}, \xi_{0}\right]$ as well as over $\left[\xi_{0}, \sup \boldsymbol{I}\right]$.

- $\quad$ Since inf $\boldsymbol{I} \in \boldsymbol{a}$, the enclosure properties of $\boldsymbol{F}$ and $\boldsymbol{P}$ proves that $R_{n}\left(f, \xi_{0}\right)(\inf \boldsymbol{I})$ belongs to $\boldsymbol{F}(\boldsymbol{a})-$ $\boldsymbol{P}\left(\boldsymbol{a}-\boldsymbol{x}_{\mathbf{0}}\right)=\boldsymbol{\Delta}_{\boldsymbol{a}}$, which is a sub-interval of $\boldsymbol{\Delta}$ (we recall that on Line 9 , " $\vee$ " denotes the "join" operator on intervals). Similarly, we prove that we have $R_{n}\left(f, \xi_{0}\right)(\sup \boldsymbol{I}) \in \boldsymbol{\Delta}$ and $R_{n}\left(f, \xi_{0}\right)\left(\xi_{0}\right) \in \boldsymbol{\Delta}$.

- Finally, for any $x \in \boldsymbol{I}$, we have $x \in\left[\inf \boldsymbol{I}, \xi_{0}\right]$ or $x \in\left[\xi_{0}, \sup \boldsymbol{I}\right]$, so combining the monotonicity of $R_{n}\left(f, \xi_{0}\right)$ and the convexity of $\boldsymbol{\Delta}$ gives $R_{n}\left(f, \xi_{0}\right)(x) \in \boldsymbol{\Delta}$. 
4) Specialising generic proofs to handle usual functions: The current version of our CoqApprox library provides fully formally proved Taylor model algorithms for the following functions: constants, identity, $x \mapsto \frac{1}{x}, \sqrt{ } \cdot \frac{1}{\sqrt{\cdot}}$, exp, sin, cos.

All of these functions are $D$-finite functions, so they fit in our framework perfectly. The implementation as well as the correctness proof of each of these functions are just instantiations of the generic algorithms and proofs.

In order to add a function to our framework, the user has to provide

- the recurrence relation between the Taylor coefficients of the function in order to be able to compute the coefficients,

- the interval evaluator for the function in order to be able to provide an initial value,

- the definition of the abstract function and properties of the function and of its derivatives in order to be able to prove correct the computed Taylor model.

We detail here the example of the exponential function. Its Taylor coefficients $\left(c_{n}\right)_{n \in \mathbb{N}}$ satisfy $c_{n}=\frac{c_{n-1}}{n}$. The corresponding COQ code is thus

Definition exp_rec $(n: \mathbb{N}) c:=\operatorname{tdiv} c$ (tnat $n$ ).

where tdiv represents division and tnat an injection of integers to the type of coefficients. We note that all this is described for an abstract type of numbers. The user will then be able to instantiate the recurrence with real numbers, or floating-point numbers, or intervals, according to his or her needs.

In order to provide the generic Taylor polynomial for the exponential, we can rely on the trec1 function for firstorder recurrences, which was presented in Section III-B3:

Definition T_exp y0 $\mathrm{n}:=$ trec1 exp_rec (texp y0) $\mathrm{n}$.

Notice that one of the arguments passed to trec1 is (texp y0), the value of the exponential at y0. Hence the necessity of having an evaluator for the function in order to "initialise" the computation.

In order to provide an $n^{\text {th }}$-order Taylor model for exp over interval $\boldsymbol{I}$, an $n^{\text {th }}$-order Taylor polynomial around $\boldsymbol{x}_{\mathbf{0}}$ is combined with an enclosure of the Taylor-Lagrange remainder as computed by Algorithm 1 (named Ztech in the code). The Taylor model that is obtained is an instance of the rpa structure described in section II:

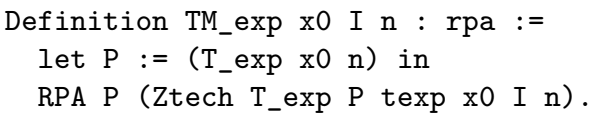

Note that we have omitted a precision argument for readability in all the above COQ definitions. This argument allows the user to set the desired precision of each computation.

As regards to proofs, the generic theorems of correctness apply to Taylor models with interval coefficients. The correctness is proved with respect to functions from the Reals standard library. The properties required for the correctness concern

- the compatibility of the interval function with the real function,

- the appropriate behaviour on the NaN value,

- the compatibility between the function and the recurrence used to compute the Taylor coefficients.

In order to ease the verification of this latter property in future versions of the library, we expect to benefit from on-going formalisation efforts not only to make the Reals library more generic but also to formalise the DDMF, ${ }^{3}$ as undertaken by the teams Toccata and Specfun from Inria, as well as the Coquelicot project. ${ }^{4}$

Once all these properties are provided, we get the proof of correctness of our algorithm by simply applying the generic theorem.

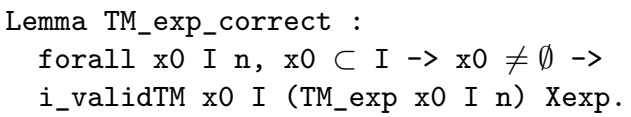

This theorem now states that the computed Taylor model TM_exp is a valid model (in the sense of Definition 1) of Xexp, which is the exponential function defined in the standard library of COQ, lifted from $\mathbb{R}$ to $\overline{\mathbb{R}}$.

Available functions and current restrictions: As stated before, we were able to implement and prove correct TMs for constant functions, identity function, $x \mapsto \frac{1}{x}$, $x \mapsto \sqrt{x}, x \mapsto \frac{1}{\sqrt{x}}$, exp, sin, cos. For these functions, all the necessary ingredients were already available in the Coq.Interval library and the Reals library. However, other functions require more work in order to have an associated Taylor model.

For example, the logarithm function is available in the Reals library, but we are not able to compute a Taylor model as the interval evaluator is not yet implemented in Coq.Interval.

Another special case is the tangent function. It is not a $D$-finite function. Its ordinary differential equation is not linear. So we cannot describe the Taylor coefficients of the tangent using a linear recurrence relation. However, we may describe them with a non-linear recurrence relation. Since our generic framework and in particular Algorithm 1 is not restricted to Taylor polynomials given by linear recurrence relations, we could implement tangent in this way. The other option is to deal with $\tan (x)$ as $\frac{\sin x}{\cos x}$, which can be handled through the Taylor models algorithms for composite functions, but yields a much slower algorithm.

\section{TAylor Models for Composite Functions}

In order to provide Taylor models for the addition, multiplication, composition and division of two functions

\footnotetext{
${ }^{3}$ The Dynamic Dictionary of Mathematical Functions, available at the URL http://ddmf.msr-inria.inria.fr/

${ }^{4}$ URL: http://coquelicot.saclay.inria.fr/
} 
we do not use a Taylor expansion (as for the base functions) but we introduce an arithmetic on Taylor models by providing a specific algorithm for each of these operations. The main reason for this choice is to ensure tighter bounds on the error estimated for the Taylor model.

The algorithms and proofs we use for these operations on Taylor models closely follow those described in [1].

\section{A. Mathematical setup}

Consider two Taylor models $\left(\boldsymbol{P}_{1}, \boldsymbol{\Delta}_{1}\right)$ and $\left(\boldsymbol{P}_{2}, \boldsymbol{\Delta}_{2}\right)$ of $n^{\text {th }}$-order, approximating $f_{1}$ and $f_{2}$ over the interval $\boldsymbol{I}$. We define the following operations on these Taylor models and obtain in each case an $n^{\text {th }}$-order Taylor model.

Addition: $\left(\boldsymbol{P}_{1}, \boldsymbol{\Delta}_{1}\right) \oplus\left(\boldsymbol{P}_{2}, \boldsymbol{\Delta}_{2}\right):=\left(\boldsymbol{P}_{1}+\boldsymbol{P}_{2}, \boldsymbol{\Delta}_{1}+\boldsymbol{\Delta}_{2}\right)$;

Multiplication: $\left(\boldsymbol{P}_{1}, \boldsymbol{\Delta}_{\mathbf{1}}\right) \otimes\left(\boldsymbol{P}_{2}, \boldsymbol{\Delta}_{2}\right):=(\boldsymbol{P}, \boldsymbol{\Delta})$ where $\boldsymbol{P}:=\left(\boldsymbol{P}_{1} \times \boldsymbol{P}_{2}\right) \leqslant n$ and $\boldsymbol{\Delta}:=\operatorname{eval}\left(\left(\boldsymbol{P}_{1} \times \boldsymbol{P}_{2}\right)_{>n}\right)+$ $\left(\operatorname{eval}\left(\boldsymbol{P}_{1}\right) \times \boldsymbol{\Delta}_{2}\right)+\left(\operatorname{eval}\left(\boldsymbol{P}_{2}\right) \times \boldsymbol{\Delta}_{1}\right)+\left(\boldsymbol{\Delta}_{1} \times \boldsymbol{\Delta}_{2}\right)$.

In the above definition $(\boldsymbol{Q})_{\leqslant n}$ denotes the polynomial (with interval coefficients) containing all the monomials of $\boldsymbol{Q}$ up to degree $n$, and $(\boldsymbol{Q})_{>n}$ those of higher degree; $\operatorname{eval}(\boldsymbol{Q})$ is the evaluation of polynomial $\boldsymbol{Q}$ over interval $\boldsymbol{I}$.

As regards the composition of two functions $f_{1} \circ f_{2}$, the Taylor model algorithm is essentially the evaluation of the polynomial corresponding to $f_{1}$ in the Taylor model of $f_{2}$. This is accomplished by relying on the addition and multiplication of Taylor models. The error is composed of the error that results from this evaluation and the error of the Taylor model of $f_{1}$.

We also define the inverse of a Taylor model and the division of two Taylor models. We compute a Taylor model for $\frac{1}{f(x)}$ by using the Taylor model algorithm for the composition $\left(x \mapsto \frac{1}{x}\right) \circ f$. We compute a Taylor model for $\frac{f(x)}{g(x)}$ as the multiplication of a Taylor model for $f(x)$ by a Taylor model for $\frac{1}{g(x)}$.

All the correctness theorems for addition, multiplication and composition of Taylor models (TMs) have the same form. For example, the correctness for the addition is stated as follows: if $\left(\boldsymbol{P}_{1}, \boldsymbol{\Delta}_{1}\right)$ and $\left(\boldsymbol{P}_{2}, \boldsymbol{\Delta}_{2}\right)$ are two valid TMs for functions $f_{1}$ and $f_{2}$ over $\boldsymbol{I}$, then the sum defined as above is a valid TM for $f_{1}+f_{2}$ over $\boldsymbol{I}$.

\section{B. Formal setup}

The arithmetic operations on Taylor models are implemented using the algorithms summarised in the previous section. As for the basic functions, we have generic algorithms for these operations, that are then instantiated with the type of coefficients and the type of polynomials desired. Here is the COQ code for the definition of addition:

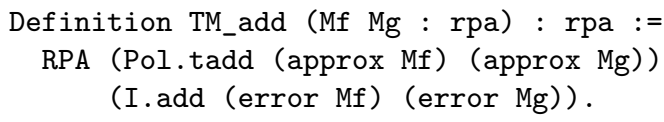

The formal proofs of correctness for addition, multiplication and composition are based on the pen-andpaper proofs that can be found in [1, Chap. 2]. The main adjustments that we have to make concern the use of $\mathrm{NaN}$ values that can be taken by the coefficients and the intervals. The pen-and-paper proofs do not deal with such values. Though, in most cases, the $\mathrm{NaN}$ values lead to trivial statements. In some situations we have to exclude the undefined values from the theorem. For example, the correctness of the multiplication of two Taylor models can only be proved for polynomials whose size is greater than zero, i.e. they must have at least one coefficient. The zero-size polynomial gives rise to a $\mathrm{NaN}$ value when evaluated. In practice, however, this is not a problem. We never output polynomials with no coefficients. The same restriction applies to the composition of two Taylor models.

As regards the correctness proofs of inverse and division, they are immediately given by applying the generic theorems asserting the correctness of composition and multiplication.

\section{BENCHMARKS}

The current version of our library is compatible with both Maxime Dénès' native-coq branch ${ }^{5}$ of COQ [11] and current version of COQ (version $8.4 \mathrm{pl} 2$ ). For evaluating the performances of our library, we rely on the nativecoq version, which features native machine integers and a compilation to fast and native OCaml code.

A state-of-the-art implementation of univariate Taylor Models is available in the Sollya tool [12], developed by Mioara Joldeş et al. and written in C, but the correctness of this implementation is not formally proved. Roughly speaking, Sollya represents polynomials as arrays of interval coefficients with multiple-precision floating-point bounds and relies on several $\mathrm{C}$ libraries such as MPFR or GMP.

Table II gives the timing and the quality of the approximation obtained for a selection of functions. A laptop based on a Intel ${ }^{\circledR}$ Core $^{\mathrm{TM}}$ i7-3720QM processor clocked at $2.60 \mathrm{GHz}$ with $8 \mathrm{~GB}$ of RAM, running GNU/Linux 3.8.13100.fc17.x86_64, the 2013-03-15 version of native-coq with OCaml 3.12.1, and Sollya 4.0 has been used.

In particular, the computations that are performed with Sollya rely on the taylorform() function. As regards the computations performed with COQ, we use the instantiation of our hierarchy with polynomials as lists (with linear access time) gathering interval coefficients with floating-point bounds, themselves built upon the machineefficient big integers that are provided by the BigZ library of COQ.

The first column of Table II gives the function and the interval $\boldsymbol{I}$. In these experiments, we have chosen to develop the Taylor models at the middle of the interval, that is $\boldsymbol{x}_{\mathbf{0}}:=[c, c]$ where $c:=\frac{1}{2}(\inf \boldsymbol{I}+\sup \boldsymbol{I})$. This column also gives the order of the TM, and the working precision for floating-point operations in radix 2 .

The three subsequent columns give the timing for computing the respective Taylor model, and the ratio

\footnotetext{
${ }^{5}$ URL: https://github.com/maximedenes/native-coq
} 
Table II. Benchmarks for our library on Taylor Models

\begin{tabular}{|c|c|c|c|c|c|c|}
\hline & \multicolumn{3}{|c|}{ Execution time } & \multicolumn{3}{|c|}{ Approximation error } \\
\hline & $\mathrm{COQ}$ & Sollya & ratio & naive $\mathrm{COQ}$ & $\mathrm{COQ}$ & Sollya \\
\hline $\begin{array}{l}f(x)=e^{x} \\
I=[2,4] \\
\text { order }=80 \\
\text { prec }=500\end{array}$ & $0.174 \mathrm{~s}$ & $0.092 \mathrm{~s}$ & 1.9 & $1.52 \cdot 2^{-396}$ & $1.14 \cdot 2^{-397}$ & $1.14 \cdot 2^{-397}$ \\
\hline $\begin{array}{l}f(x)=\sin x \\
\boldsymbol{I}=[-1,1] \\
\text { order }=80 \\
\text { prec }=500\end{array}$ & $0.146 \mathrm{~s}$ & $0.092 \mathrm{~s}$ & 1.6 & $1.79 \cdot 2^{-402}$ & $1.79 \cdot 2^{-402}$ & $1.79 \cdot 2^{-402}$ \\
\hline $\begin{array}{l}f(x)=\frac{1}{x} \\
\boldsymbol{I}=[1,3] \\
\text { order }=100 \\
\text { prec }=125\end{array}$ & $0.022 \mathrm{~s}$ & $0.165 \mathrm{~s}$ & 0.13 & $1 \cdot 2^{0}$ & $1 \cdot 2^{-101}$ & $1 \cdot 2^{-101}$ \\
\hline $\begin{array}{l}f(x)=\sqrt{x} \\
I=[1,3] \\
\text { order }=100 \\
\text { prec }=125\end{array}$ & $0.037 \mathrm{~s}$ & $0.169 \mathrm{~s}$ & 0.22 & $1.98 \cdot 2^{-12}$ & $1.60 \cdot 2^{-112}$ & $1.60 \cdot 2^{-112}$ \\
\hline $\begin{array}{l}f(x)=\frac{1}{\sqrt{x}} \\
\text { (as a basic function) } \\
\boldsymbol{I}=[1,3] \\
\text { order }=100 \\
\text { prec }=125\end{array}$ & $0.029 \mathrm{~s}$ & $0.424 \mathrm{~s}$ & 0.068 & $1.80 \cdot 2^{-5}$ & $1.27 \cdot 2^{-105}$ & $1.27 \cdot 2^{-105}$ \\
\hline $\begin{array}{l}f(x)=e^{x} \sin x \\
\boldsymbol{I}=\left[-\frac{3}{2}, \frac{3}{2}\right] \\
\text { order }=50 \\
\text { prec }=500\end{array}$ & $0.497 \mathrm{~s}$ & $0.048 \mathrm{~s}$ & 10 & $1.94 \cdot 2^{-166}$ & $1.94 \cdot 2^{-166}$ & $1.94 \cdot 2^{-166}$ \\
\hline $\begin{array}{l}f(x)=e^{x} \sin x \\
\boldsymbol{I}=\left[-\frac{3}{2}, \frac{3}{2}\right] \\
\text { order }=100 \\
\text { prec }=500\end{array}$ & $1.010 \mathrm{~s}$ & $0.306 \mathrm{~s}$ & 3.3 & $1.63 \cdot 2^{-423}$ & $1.63 \cdot 2^{-423}$ & $1.63 \cdot 2^{-423}$ \\
\hline $\begin{array}{l}f(x)=e^{1 / \cos x} \\
\boldsymbol{I}=[0,1] \\
\text { order }=50 \\
\text { prec }=100\end{array}$ & $6.378 \mathrm{~s}$ & $0.095 \mathrm{~s}$ & 67 & $1.46 \cdot 2^{-23}$ & $1.45 \cdot 2^{-41}$ & $1.45 \cdot 2^{-41}$ \\
\hline $\begin{array}{l}f(x)=e^{1 / \cos x} \\
\boldsymbol{I}=[0,1] \\
\text { order }=100 \\
\text { prec }=100\end{array}$ & $52.92 \mathrm{~s}$ & 0.653 & 81 & $1.97 \cdot 2^{-49}$ & $1.99 \cdot 2^{-89}$ & $1.98 \cdot 2^{-89}$ \\
\hline $\begin{array}{l}f(x)=\frac{\sin x}{\cos x} \\
\boldsymbol{I}=[-1,1] \\
\text { order }=50 \\
\text { prec }=100\end{array}$ & $1.228 \mathrm{~s}$ & $0.083 \mathrm{~s}$ & 15 & $1.06 \cdot 2^{14}$ & $1.66 \cdot 2^{-32}$ & $1.10 \cdot 2^{-52}$ \\
\hline $\begin{array}{l}f(x)=\frac{\sin x}{\cos x} \\
\boldsymbol{I}=[-1,1] \\
\text { order }=100 \\
\text { prec }=100\end{array}$ & $11.15 \mathrm{~s}$ & $0.570 \mathrm{~s}$ & 20 & $1.45 \cdot 2^{26}$ & $1.12 \cdot 2^{-64}$ & $1.82 \cdot 2^{-96}$ \\
\hline $\begin{array}{l}f(x)=\frac{1}{\sqrt{x}} \\
\text { (as a composite function) } \\
\boldsymbol{I}=[1,3] \\
\text { order }=100 \\
\text { prec }=125\end{array}$ & $37.683 \mathrm{~s}$ & $0.424 \mathrm{~s}$ & 89 & $1.98 \cdot 2^{-12}$ & $1.27 \cdot 2^{-105}$ & $1.27 \cdot 2^{-105}$ \\
\hline
\end{tabular}

Time $_{\text {COQ }} /$ Time $_{\text {Sollya. }}$ We thus notice that these timings are of the same order of magnitude for all basic functions (the shortest ones being printed in bold). We also notice that on these examples ranging up to degree 100, the COQ timings for composite functions are only 3 to 89 times slower than the Sollya implementation, which is reasonable, given that the $\mathrm{COQ}$ implementation is being executed in a trusted environment with restricted computing power.

The last three columns give the magnitude of the error interval that takes into account both the method error and the rounding errors involved in the approximation. To sum up, it corresponds to the value $\max \left\{\left|\inf \boldsymbol{\Delta}^{\prime}\right|,\left|\sup \boldsymbol{\Delta}^{\prime}\right|\right\}$ rounded towards $+\infty$, where $\boldsymbol{\Delta}^{\prime}$ has been computed to satisfy Definition 2. The column "naive COQ" corresponds to our former implementation of Taylor Models for basic functions that simply relies on a naive enclosure of the Taylor-Lagrange remainder, while the column "COQ" corresponds to the optimised implementation, based on Algorithm 1. For the sake of readability, all these bounds are written as a power of 2 multiplied by a decimal number between 1 and 2 .

First, we can notice that the optimisation due to Algorithm 1 significantly improves the bounds for some basic functions such as the reciprocal function or the square root, as well as for the composite functions that involve division. Then, we notice that for each basic function, the error bounds computed by COQ, resp. Sollya, have exactly the same order of magnitude. As regards the composite functions, the same remark applies, except that Sollya's bounds for $\tan x=\frac{\sin x}{\sin x}$ are tighter than those of COQ. We expect that this latter difference is explained by a difference in the implemented algorithms, since our tangent is seen as a composite function. Finally, the availability of a Taylor model algorithm for $x \mapsto \frac{1}{\sqrt{x}}$ seen as a basic function allows one to check, as expected, that this algorithm is much faster than considering $x \mapsto \frac{1}{\sqrt{x}}$ as a composite function. Yet as regards the provided error bounds, we do not notice any difference for this particular function.

\section{Related WorkS}

To our knowledge, the first formalisation of multivariate Taylor models is described in [13]. It relies on exact real arithmetic, but no formal proof is available. A formallyproved implementation of univariate Taylor models in the PVS proof assistant is presented in [14]. However, the Taylor models are defined in an ad-hoc way for few functions and with 6 as maximal degree. Another formally-proved implementation is described in [15]. The coefficients are axiomatised floating-point numbers so the formalisation is not directly executable. Finally, more recently, a formallyproved library to solve nonlinear inequalities using Taylor approximations in HOL LIGHT has been proposed in [16]. The focus is on small-degree, multivariate polynomials while in our applications we are mostly interested in highdegree (up to 90 in some cases) univariate Taylor models.

\section{Conclusion And Future Work}

Our initial interest in developing a certified library that manipulates Taylor models comes from a long term project to validate the worst cases for correct rounding of elementary functions. A first step in this validation is to be able to certify the quality of an approximation. Given a function $f$, an approximation $P$, a bound $\epsilon$ and an interval $\boldsymbol{I}$, we would like to derive automatically within COQ that $|f(x)-P(x)|<\epsilon$ for $x \in \boldsymbol{I}$. What we have achieved with our library of Taylor models is to be able to prove automatically something like $\left|f(x)-T M_{f}(x)\right|<\epsilon_{1}$ for $x \in \boldsymbol{I}$. For this work, we have followed a "prototype and prove" strategy. In the first stage, we have used the COQ system as a mere programming language to implement our library. This is the prototyping phase that was described in [4]. We ended up with a library that was worth proving. The second stage (and the most time-consuming one) was 
to formally verify this library. The resulting library has the following characteristics:

- It is generic. Thanks to our design based on modules, we can easily and independently change various aspects of the library (representation of the polynomials, representation of coefficients, representation of intervals). Also, we have taken a great care to develop proofs that are as generic as possible.

- It is efficient enough. We have made a number of tests that seems to indicate that we are only one order of magnitude slower than the implementation of Sollya.

- It has been formally verified. We have correctness proofs for the operations on Taylor models (namely $+, \times, \circ, \div)$ as well as for the basic functions $x \mapsto \frac{1}{x}$, $\sqrt{\cdot}, \frac{1}{\sqrt{\cdot}}, \exp$, sin and cos.

- It returns sharp bounds. In particular, we have performed a number of tests to demonstrate the impact of an optimisation of our algorithms (based on a result from Roland Zumkeller's thesis).

The library could still be further improved. Some interesting basic functions are still missing like arctan, tan and log. Also, Karatsuba algorithm could be implemented in order to multiply two Taylor models more efficiently. Nevertheless, our next priority is to complete our validation by providing an automatic tool within COQ to bound a polynomial on an interval. Different standard techniques exist that have already been applied in a formal setting, such as sum of squares [17] or Bernstein polynomials [18]. Combined with our Taylor models which prove $\left|f(x)-T M_{f}(x)\right|<\epsilon_{1}$ for $x \in \boldsymbol{I}$, we could also derive that $\left|T M_{f}(x)-P(x)\right|<\epsilon_{2}$. Then, a simple application of the triangle inequality would lead to the expected validation $|f(x)-P(x)|<\epsilon$ for a judicious choice of $\epsilon_{1}$ and $\epsilon_{2}$.

\section{REFERENCES}

[1] M. Joldeş, "Rigourous Polynomial Approximations and Applications," Ph.D. dissertation, ENS Lyon, 2011. [Online]. Available: http://tel.archives-ouvertes.fr/tel-00657843/en/

[2] K. Makino and M. Berz, "Taylor Models and Other Validated Functional Inclusion Methods," International Journal of Pure and Applied Mathematics, vol. 4, no. 4, pp. 379-456, 2003.

[3] K. Makino, "Rigorous Analysis of Nonlinear Motion in Particle Accelerators," Ph.D. dissertation, Michigan State University, East Lansing, Michigan, USA, 1998.

[4] N. Brisebarre, M. Joldes, É. Martin-Dorel, M. Mayero, J.-M. Muller, I. Paşca, L. Rideau, and L. Théry, "Rigorous Polynomial Approximation Using Taylor Models in Coq," in NFM'12, ser. LNCS, vol. 7226. Springer, 2012, pp. 85-99.

[5] G. Gonthier, A. Mahboubi, and E. Tassi, "A Small Scale Reflection Extension for the Coq system," INRIA, Research Report RR-6455, 2008. [Online]. Available: http://hal.inria.fr/ inria-00258384/en/

[6] G. Melquiond, "Floating-point arithmetic in the Coq system," Information and Computation, vol. 216, pp. 14-23, 2012.

[7] M. Mayero, "Formalisation et automatisation de preuves en analyses réelle et numérique," Ph.D. dissertation, Paris VI University, France, 2001.
[8] É. Martin-Dorel, "Contributions to the Formal Verification of Arithmetic Algorithms," Ph.D. dissertation, ENS Lyon, Sep. 2012. [Online]. Available: http://tel.archives-ouvertes.fr/ tel-00745553/en/

[9] S. Chevillard, J. Harrison, M. Joldeş, and C. Lauter, "Efficient and accurate computation of upper bounds of approximation errors," Theoretical Computer Science, vol. 16, no. 412, pp. 1523-1543, 2011.

[10] R. Zumkeller, "Global Optimization in Type Theory," Ph.D. dissertation, École polytechnique, France, 2008. [Online]. Available: http://alacave.net/ roland/FormalGlobalOpt.pdf

[11] M. Boespflug, M. Dénès, and B. Grégoire, "Full Reduction at Full Throttle," in CPP, ser. LNCS, vol. 7086. Springer, 2011, pp. 362-377.

[12] S. Chevillard, M. Joldeş, and C. Lauter, "Sollya: An Environment for the Development of Numerical Codes," in Mathematical Software - ICMS 2010, ser. LNCS, vol. 6327. Springer, September 2010, pp. 28-31.

[13] R. Zumkeller, "Formal Global Optimisation with Taylor Models," in IJCAR, ser. Lecture Notes in Computer Science, U. Furbach and N. Shankar, Eds., vol. 4130. Springer, 2006, pp. 408-422.

[14] F. Cháves, "Utilisation et certification de l'arithmétique d'intervalles dans un assistant de preuves," Ph.D. dissertation, ENS Lyon, Sep. 2007. [Online]. Available: http://tel.archives-ouvertes.fr/tel-00177109/en/

[15] P. Collins, M. Niqui, and N. Revol, "A Validated Real Function Calculus," Mathematics in Computer Science, vol. 5, no. 4, pp. 437-467, 2011.

[16] A. Solovyev and T. C. Hales, "Formal Verification of Nonlinear Inequalities with Taylor Interval Approximations," in NFM'13, ser. LNCS, vol. 7871. Springer, 2013, pp. 383-397.

[17] J. Harrison, "Verifying Nonlinear Real Formulas Via Sums of Squares," in TPHOLs, ser. LNCS, vol. 4732, 2007, pp. 102-118.

[18] C. Muñoz and A. Narkawicz, "Formalization of Bernstein Polynomials and Applications to Global Optimization," Journal of Automated Reasoning, pp. 1-46, 2012. 